Final peer reviewed manuscript accepted in Journal of Forensic Practice, 2017.

\title{
Negative relating styles of learning-disabled and non-learning-disabled offenders
}

\author{
Craven, R., \& Tonkin, M.
}

\begin{abstract}
Understanding the relating style of offenders is a relatively new concept in forensic psychology with research focusing on the association between relating and different categories of offenders (Newberry \& Birtchnell, 2011). 'Relating theory' is based on the premise that we are born with a predisposition to relate to others in eight primary ways, and with maturity we can achieve a competent relating style. These eight positions incorporate the four key relating objectives of 'upperness', 'lowerness', 'closeness' and 'distance' that form the basis of the interpersonal octagon. The interpersonal octagon is organised around two intersecting axes: 'close' versus 'distant' on the horizontal axis, and 'upper' versus 'lower' on the vertical axis. A blend of the horizontal and vertical states create four intermediate positions completing the octagon. 'Close' is an interactional process of seeking closeness with the desire to gain greater involvement with others, whilst individuals with a 'distant' relating style have a need for separation and self-efficacy (Birtchnell, 1994). 'Upper' is a multifaceted relating objective that allows an individual to gain a sense of superiority in relation to others, versus 'lower' where the individual has a dependent relating style (Birtchnell, 1994). A relating style that lacks versatility and competence is known as negative relating.
\end{abstract}


Final peer reviewed manuscript accepted in Journal of Forensic Practice, 2017.

Relationship status and relating styles are incorporated within actuarial and dynamic risk assessments e.g. Risk Matrix 2000 (Thornton, 2010) and Structured Assessment of Risk and Need (Home Office, 2005). Using these assessments on learning-disabled (LD) offenders can increase their risk level (Brown \& Stein, 1997) as they are less likely to have engaged in an enduring relationship due to interpersonal deficits (Day, 1994). Although relationship status is an integral part of assessing risk, limited research has focused on the offender's relating ability. Developing an understanding of the negative relating styles of LD and non-LD offenders is essential to identifying the treatment needs of these distinct groups. By increasing our knowledge of LD offenders and their associated relating styles, the theoretical underpinnings of risk assessments can be enhanced. In line with the Risk Need Responsivity approach (Bonta \& Andrews, 2007) increased knowledge of the risk and needs of LD offenders can support the development of LD-specific interventions.

The interpersonal octagon is a well-validated and accepted model for defining and classifying relating styles. The Person's Relating to Others Questionnaire (PROQ2) was designed to measure relating within the octagon and has been used in various studies to assess relating styles in psychotherapy settings (Birtchnell, 2002), with depressed patients (Birtchnell et al., 1992), and personality disorders (Birtchnell \& Shine, 2000). The shorter PROQ3 was designed to measure negative relating, as illustrated in Figure 1 and has been shown to have good internal reliability, correlating positively with other theoretically-related measures, such as the Interpersonal Checklist-Revised and the Circumplex Version of the Inventory of Interpersonal Problems (see Birtchnell et al., 2011).

\section{$<<$ Insert Figure 1 around here $>>>$}

As a theoretical structure, the interpersonal octagon has been applied to understanding the association between relating styles and offending behaviours. Shine and Birtchnell (2002) conducted a pilot study on 107 prisoners to assess the relationship between offence types and interpersonal relating using the PROQ2. The study extracted four offence categories (homicide, robbery, sex, and other violence excluding homicide). The results revealed that sexual offenders had the highest mean scores on each subscale, indicating that they had reduced abilities at forming relationships. To avoid contamination by other previous offence types, a pure sex offender group $(n=6)$ was compared to the remaining sample of offenders who had no previous or current sexual convictions $(n=69)$. The sexual offenders scored 
Final peer reviewed manuscript accepted in Journal of Forensic Practice, 2017.

significantly higher on the total score as well as the Neutral Distant (ND) and Upper Neutral (UN) subscales. These findings support previous research that shows sexual offenders to have increased interpersonal deficits, which has the potential to influence treatment needs (Marshall, 1989).

Further, Newberry and Birtchnell (2011) examined the relationship between negative relating styles (as measured by the PROQ3) and offence types, compared the relating styles of 923 incarcerated male offenders to 142 non-offending males from various regions within the United Kingdom (UK). Four pure offence categories were created for the offender sample that consisted of homicide, violent, sexual, and dishonest offences. The study concluded that the ND and Lower Close (LC) subscales of the PROQ3 were most associated with criminality. The offender group scored significantly higher on the ND scale in comparison to the non-offender group. This is not surprising as the ND scale is characterised by a lack of concern for others; a trait associated with criminality (Newberry \& Birtchnell, 2011). As predicted, the offender group also scored significantly higher than the non-offender sample on the LC scale that correlates highly with almost all of the DSM-IV personality disorder types (Birtchnell \& Shine, 2000). The study found that sexual and dishonest offenders had significantly higher scores on the LC scale compared to the non-offenders, indicating that fear of rejection and disapproval was a feature within these offender groups. The pure dishonest offenders had the highest mean total indicating that negative relating is particularly pertinent for this group. The non-offender sample scored significantly higher on the UN scale compared to the offender group indicating that the offenders in the study were less inclined to present in a controlling manner.

\section{Relating style amongst LD offenders}

No published studies have specifically examined the relating styles of LD offenders. Thus, any understanding of the association between negative relating and offending within the LD population, and how relating styles might differ between LD and non-LD offenders is limited. Newberry and Shuker (2011) found that prisoners with lower levels of intellectual ability had increased psychological instability and greater treatment needs. Such gaps in knowledge need to be addressed in order to assess whether the findings of Shine and Birtchnell (2002) and Newberry and Birtchnell (2011) can be generalised to all types of offenders regardless of intellectual ability. The majority of studies have focused on LD sexual offenders with limited research on other types of LD offenders. Despite higher recidivism 
Final peer reviewed manuscript accepted in Journal of Forensic Practice, 2017.

rates within the LD population in comparison to the general population (Griffiths et al., 1985), LD offenders remain a relatively under-researched group (Craig \& Hutchinson, 2005). It is, therefore, important to compare LD and non-LD offenders in terms of their relating styles and to assess whether an association between specific offence categories and different negative relating styles exist within the LD population.

This study compares the negative relating styles of LD and non-LD offenders, further examining the relationship between specific offence categories and different forms of negative relating within an LD sample. The research sought to test whether the findings of Shine and Birtchnell (2002) and Newberry and Birtchnell (2011) could be applied to LD offenders. Three hypotheses were examined in this study:

1) Given the increased comorbidity issues associated with LD individuals, it is expected that such characteristics will be heightened within the LD offender population (Cooper et al., 2007). Thus, LD offenders will show higher scores on the LC scale in comparison to the non-LD population.

2) Given that LD offenders are known to experience higher levels of socio-affective deficits (Marshall, 1989), it was hypothesised that the LD sample would have higher scores on the Neutral Close (NC) scale compared to the non-LD offender sample.

3) Sexual offenders have been shown to have increased levels of perceived rejection and disapproval (Overholser \& Beck, 1986). These relating characteristics are featured within the LC scale, thus it was hypothesised that sexual offenders would demonstrate higher scores on the LC scale in comparison to the other three offence categories.

\section{Method}

\section{Participants}

Two groups of male offenders were recruited to the study based on their intellectual ability. The first group consisted of LD offenders $(n=18)$ detained within a low secure and locked rehabilitation hospital with a primary diagnosis of mental impairment. The second group consisted of offenders $(n=30)$ incarcerated at a Category B private male prison. At the point of admission, the offenders were assessed using the Ravens Standard Progressive Matrices and deemed to have average or above average intellectual ability. 
Final peer reviewed manuscript accepted in Journal of Forensic Practice, 2017.

Table 1 outlines the characteristics of the sample. The LD offenders had a mean age of 49.61 years $(S D=11.85)$ and were detained for an average of 7.17 years $(S D=3.65)$ in the hospital. The non-LD offender group had a mean age of 34.10 years $(S D=9.54)$ and were incarcerated for an average of 1.53 years $(S D=.68)$. To assess whether offence types were associated with different forms of negative relating (regardless of intellectual ability), participants were placed into one of four categories (homicide, sexual offences, violence and robbery) based on their index offence as outlined in Table 1. Homicide offenders were those with a conviction for murder, attempted murder, and manslaughter. Sexual offenders had convictions for buggery, rape, sexual assault, indecent assault, unlawful sexual intercourse, and other miscellaneous sexual offences. Violent offenders were those with convictions for assault, wounding, actual bodily harm, grievous bodily harm, arson, kidnap, and drunk and disorderly behaviour. The robbery category was a pure offence category, consisting solely of robbery offences. Access to information relating to previous convictions for the sample was not possible due to organisational restrictions from the prison; therefore the study was unable to extract pure offence categories for analysis.

\section{Materials}

The study used the PROQ3, which is a 48-item questionnaire (6 items per octant scale). Each octant has five negative items and one positive item; only the negative items are scored as the positive items are only included to alleviate the overall negative tone of the questionnaire. The responses are 'Nearly always true', 'Quite often true', 'Sometimes true', and 'Rarely true' which carry a score of 3, 2, 1 and 0 respectively. The questionnaire has a maximum score of 120 and each octant has a maximum score of 15 . Increased scores indicated greater deficits in relating competence.

As the PROQ3 has never been used with LD offenders, it was adapted to accommodate for their cognitive deficits. A working group was established to adapt it into an accessible format using visual aids, and an additional glossary to enhance understanding. The adapted material was reviewed by a registered speech and language therapist and a pilot was undertaken with two LD participants to further assess the appropriateness of the adapted information. No problems were highlighted in the pilot and therefore this data was included in the main analysis 
Final peer reviewed manuscript accepted in Journal of Forensic Practice, 2017.

Cronbach's alpha was used to assess the internal consistency and reliability of the PROQ3 data. Missing data was managed using the pairwise deletion method. The eight scales and the total score yielded good internal reliabilities for the LD sample with the following alpha coefficients obtained (Total .76; UN .69; Upper Close (UC) .67; NC .69; LC .74; Lower Neutral (LN) .77; Lower Distant .78; ND .76; and Upper Distant (UD) .71). The PROQ3 achieved a similar, satisfactory level of internal consistency in the non-LD sample (Total .79; UN .79; UC .74; NC .76; LC .71; LN .76; Lower Distant .77; ND .77; and UD .80).

\section{Design and procedure}

An independent groups design was used and both groups were administered the same questionnaire (PROQ3), with the LD offenders completing the adapted version.

All LD offenders within the hospital were considered for the study and the Responsible Clinician (RC) assessed their suitability for inclusion, with five LD offenders being excluded based on RC advice. The consent process was undertaken by a registered nurse to ensure the LD offenders did not feel implicitly coerced to participate in the study. After the LD sample had consented, the Mental Health Administrator provided the following details: date of birth (DOB), length of detainment at the hospital and conviction. The researcher administered the adapted PROQ3 to the LD offenders in the presence of a nurse so capacity and mental state was assessed throughout.

For the non-LD prison sample, the PROQ3 was completed and consent was obtained at the point of admission. Therefore it was not necessary to repeat the questionnaire. The data extraction focused on prisoners entering a prison Therapeutic Community between September 2011 and June 2014. The data for every $10^{\text {th }}$ prisoner listed alphabetically was extracted until a sample of thirty was collated. After the sample was defined, a member of the psychology team at the prison provided the following information relating to index offence, date of admission and DOB for the sample.

\section{Ethics}

Ethical approval was obtained from the research ethics committee at Birmingham City University, Social Care Institute for Excellence, and the Prison Service. A dual approach was applied to ensure the issue of capacity to consent was obtained appropriately for the LD group. 
Final peer reviewed manuscript accepted in Journal of Forensic Practice, 2017.

\section{Results}

Comparing the $L D$ and non-LD samples in terms of their PROQ3 scores.

The relating styles across the eight PROQ3 sub-scales and the total score for the LD and nonLD offender group were compared using independent samples t-tests with a Bonferroni adjusted alpha of .006. These findings are summarised in Table 2. Statistically significant differences between the LD and non-LD groups were identified in the total score as well as four of the eight subscales. The LD sample achieved significantly higher scores on the total score $(t=5.03, p<.001)$ and the UN $(t=4.97, p<.001)$, UC $(t=4.09, p<.001)$, NC $(t=$ 4.22, $p<.001)$, and Lower Distant $(t=4.73, p<.001)$ subscales compared to the non-LD sample (thereby indicating greater deficits in relating competence). Although the LD offenders obtained higher scores on the remaining subscales (LC, ND, UD, \& LN), these differences were non-significant.

\section{$<<$ INSERT Table 2 AROUND HERE $>>$}

\section{Comparing index offence categories in terms of their PROQ3.}

A one-way analysis of covariance (ANCOVA) was conducted to compare the four different offence types in terms of their relating styles, whilst controlling for the presence of a LD and length of detainment. The ANCOVA was followed up with post-hoc comparisons using independent samples t-tests with a Bonferroni adjusted alpha of .006. These findings are summarised in Table 3. Sexual offenders had the lowest overall total score in comparison to the other offence categories. The ND subscale yielded the highest mean total compared to the other seven subscales. Whilst controlling for level of functioning (LD vs non-LD), statistically significant differences were identified in six of the eight subscales including the total score. The robbery offenders achieved significantly higher scores on the total score $(F=$ $14.40, p<.001)$ and the following four subscales: $\mathrm{UN}(F=17.74, p<.001)$, NC $(F=9.93, p$ $<.01)$, ND $(F=7.46, p<.01)$ and UD $(F=8.01, p<.01)$. Violent offenders scored significantly higher on the UC $(F=9.96, p<.01)$ and Lower Distant $(F=8.10, p<.01)$ subscales in comparison to the other offence types. There were no significant differences between the offence categories on the total score, or any of the PROQ3 subscales when length of detainment was included as a covariate. 
Final peer reviewed manuscript accepted in Journal of Forensic Practice, 2017.

\section{$<<$ INSERT Table 3 AROUND HERE $>>$}

\section{Discussion}

The findings of the study were consistent with previous research which indicated that the ND scale of the PROQ3 is associated with criminality. Within this study, the ND scale obtained the highest mean scores for the offence categories, highlighting that all offenders endorsed the negative characteristics associated with ND, such as lack of concern for others, suspiciousness and limited capacity for involvement with others.

Whilst the LD offenders obtained higher scores on the LC scale in comparison to the non-LD offender group, this difference was not statistically significant. These findings are consistent with previous research that has shown that LD offenders have increased deficits in attachment and socialisation (Steptoe et al., 2006). The LC scale was the second highest mean ranked scale of the PROQ3 for the non-LD offenders group indicating that fear of rejection and disapproval was experienced by both groups. As the LC scale is highly correlated with psychopathology, these results are not surprising as increased scores on the LC scale are more prevalent amongst psychiatric in-patients and imprisoned offenders due to their dissociation from society (Birtchnell et al., 2009). Regardless of the cognitive ability of the offenders in this sample, the LC characteristics of disapproval and low self-worth were experienced.

Consistent with the hypotheses of this study, the findings demonstrated that LD offenders had significantly higher scores on the NC scale of the PROQ3 than the non-LD offenders, indicating greater deficits in this aspect of relating style. Research has shown that LD offenders experience high levels of fear around separation and being isolated from others (Marshall, 1989), therefore a significant difference between the LD and non-LD offenders groups on this scale is not surprising.

High scores on the UN scale indicate a self-assured, arrogant and dominating relating style which closely resembles the hostile-dominant component of psychopathy (Daffern et al., 2012). Interestingly, the LD offenders demonstrated statistically higher scores on this scale than the non-LD offenders. The UN scale has been shown to correlate more readily with males in the general population than offenders (Newberry \& Birtchnell, 2011); hence it is questionable whether the UN scale within the LD population is an accurate measurement of 
Final peer reviewed manuscript accepted in Journal of Forensic Practice, 2017.

this relating style. Elevated scores on the UN scale could have resulted from the LD offenders being more honest with their responses; alternatively it served as a protective factor (Newberry \& Birtchnell, 2011). Regardless of the conclusion, the results highlight the need to re-evaluate the accuracy of the UN scale.

In contrast to previous research, the sexual offender group within this sample had the lowest overall mean compared to the remaining offence groups. The results indicated that robbery offenders had increased relating deficits, with statistically significant differences on the total scores as well as four of the scales: UN, NC, ND and UD. These results are consistent with the findings of Newberry and Birtchnell (2011).

The overall results of the study suggest that LD offenders have increased relating deficits compared to the non-LD offenders, supporting the assertion that lower functioning offenders have more complex treatment needs (Newberry \& Shuker, 2011). The LD offenders demonstrated increased scores on the Lower Distant scale, reflecting a negative relating style associated with vulnerability, obedience and a tendency to withdraw from social situations. Equally, the LD offenders demonstrated significantly higher scores on the UC scales representing a relating style that is possessive, intrusive and associated with a fear of abandonment. It is well documented that LD offenders have significant interpersonal deficits (Marshall, 1989), hence these results are consistent with previous research.

Although further research would assist in the validation of the UN scale of the PROQ3, this preliminary study has increased the potential use of the PROQ3 within a wider range of forensic populations. A significant attribute of the PROQ3 is that it reflects an individual's current relating style and is not reliant on a retrospective account of attachment. As LD offenders have increased deficits with their working memory, the PROQ3 could alleviate the potential cognitive pressure of having to recall past events, hence the appropriateness of utilising the questionnaire with LD populations.

The appropriateness of adapting mainstream assessments for the LD population is an ongoing area of debate. Within the study, the concepts were simplified and explained to the LD participants. By their responses, it was apparent that they understood the questionnaire, however the possibility of procedural contamination remains. The current sample yielded good internal reliabilities on the eight scales of the PROQ3 with higher alpha coefficients 
Final peer reviewed manuscript accepted in Journal of Forensic Practice, 2017.

observed in comparison to the psychotherapy sample used to validate the PROQ3 (Birtchnell et al., 2013). Such findings indicate that the PROQ3 has the potential to be used with the LD offenders.

\section{Limitations}

Due to the restricted number of LD offender participants, the current sample cannot be seen as a representation of LD offenders; therefore further research is required to assess the effectiveness of using the adapted PROQ3 with this population. A larger sample size would have allowed for a 2 way ANOVA to be used which could potentially reveal interaction effects that exist between the LD and non-LD groups, as well as the possibility of creating pure offence categories.

Given the organisational restrictions, the sample were not screen for the presence of personality disorders (PD), or have information relating to previous convictions, length of detainment, and previous engagement in psychological intervention. These could have impacted on the participants' relating styles. Research has shown that PROQ3 scores can improve with treatment and time, which could affect the reliability of the results. It is recommended that such factors are included in future research aiming to examine the relationship between PROQ3 scores and offending.

\section{Conclusions}

All the LD offenders in the study had a primary diagnosis of mental impairment and the study compared the relating styles of LD and non-LD offenders, as well as examining the relationship between relating style and offending behaviour. Following adaptation, the PROQ3 appeared to be an accessible measure for individuals with intellectual disability; although it should be noted that some LD offenders required increased support. Therefore, further research is required to assess the use of the PROQ3 for individuals with varying degrees of intellectual functioning. The current findings suggest that cognitive functioning is associated with increased interpersonal deficits, as the LD offenders had elevated scores on all the PROQ3 scales (in comparison to the non-LD offenders), thereby indicating greater treatment need. In order to rectify some of the methodological limitations of the study, a larger sample of LD offenders should be included in future research. Aspects such as personality disorder, previous conviction, length of detainment, and engagement in treatment should also be factored into the analysis. Despite its limitations, the study provided some 
Final peer reviewed manuscript accepted in Journal of Forensic Practice, 2017.

preliminary insight into the negative relating styles of LD offenders and contributes to the discussion regarding if, and how, interventions should be modified for LD offender populations in order to better address their treatment needs.

\section{Implications for practice}

- The need for research to focus on evaluating the treatment needs of lower functioning offenders in order to aid the development of LD-specific interventions.

- The need to evaluate the appropriateness of adapting mainstream offence focused programmes for the LD population, given that there are potentially different treatment needs between these two distinct groups. 
Final peer reviewed manuscript accepted in Journal of Forensic Practice, 2017.

\section{References}

Birtchnell, J. 1994. 'The interpersonal octagon: An alternative to the interpersonal circle', Human Relations, 47(5), pp. 511-529.

Birtchnell, J. 1996. How humans relate. Hove: Psychology press.

Birtchnell, J. 2002. 'Psychotherapy and the interpersonal octagon', Psychology and Psychotherapy: Theory, Research and Practice, 75(3), pp. 349-363.

Birtchnell, J., Falkowski, J., \& Steffert, B. 1992. 'The negative relating of depressed patients: A new approach', Journal of affective disorders, 24(3), pp. 165-176.

Birtchnell, J., Hammond, S., Horn, E., De Jong, C., \& Kalaitzaki, A. 2011. 'A Cross-National Comparison of a Shorter Version of the Person's Relating to Others Questionnaire', Clinical psychology \& psychotherapy, 20(1), pp. 36-48.

Birtchnell, J., \& Shine, J. 2000. 'Personality disorders and the interpersonal octagon', British Journal of Medical Psychology, 73(4), pp. 433-448.

Bonta, J., \& Andrews, D. A. 2007. 'Risk-need-responsivity model for offender assessment and rehabilitation', Rehabilitation, 6, pp. 1-22.

Brown, H., \& Stein, J. 1997. 'Sexual abuse perpetrated by men with intellectual disabilities: a comparative study', Journal of Intellectual Disability Research, 41(3), pp. 215-224.

Cooper, S. A., Smiley, E., Morrison, J., Williamson, A., \& Allan, L. 2007. Mental ill-health in adults with intellectual disabilities: prevalence and associated factors. The British Journal of Psychiatry, 190(1), pp. 27-35.

Craig, L.A. and Hutchinson, R.B., 2005. Sexual offenders with learning disabilities: Risk, recidivism and treatment. Journal of Sexual Aggression, 11(3), pp.289-304. 
Final peer reviewed manuscript accepted in Journal of Forensic Practice, 2017.

Daffern, M., Podubinski, T., \& Lee, S. 2012. 'Recent developments in the application of measures of interpersonal style to forensic practice', SITAR newsletter, 12(3). Available from: http://sitarnewsletter.weebly.com/invited-paper-ndash-daffern.html [Accessed 8th June 2014]

Day, K. 1994. 'Male mentally handicapped sex offenders', The British Journal of Psychiatry, 165(5), pp. 630-639.

Griffiths, D., Hindsburger, D., \& Christian, R. 1985. Treating developmentally handicapped sexual offenders: The York Behaviour Management Services treatment program. Psychiatric Aspects of Mental Retardation Reviews.

Home Office. 2005. Structured Assessment of Risk and Need. Unpublished document

Marshall, W. L. 1989. 'Intimacy, loneliness and sexual offenders', Behaviour Research and Therapy, 27(5), pp. 491-504

Newberry, M., \& Birtchnell, J. 2011. 'Negative relating and offense type', Journal of Criminal Psychology, 1(1), pp. 24-35.

Newberry, M., \& Shuker, R. 2011. 'The relationship between intellectual ability and the treatment needs of offenders in a therapeutic community prison', Journal of Forensic Psychiatry \& Psychology, 22(3), pp. 455-471.

Overholser, J. C., \& Beck, S. 1986. Multimethod assessment of rapists, child molesters, and three control groups on behavioral and psychological measures. Journal of Consulting and Clinical Psychology, 54(5), pp. 682.

Shine, J., \& Birtchnell, J. 2002. 'Offence typology and the interpersonal octagon: An exploratory analysis’, Criminal Behaviour and Mental Health, 12(3), pp. 227-229.

Steptoe, L., Lindsay, W. R., Forrest, D., \& Power, M. 2006. 'Quality of life and relationships in sex offenders with intellectual disability', Journal of Intellectual and Developmental Disability, 31(1), pp. 13-19. 
Final peer reviewed manuscript accepted in Journal of Forensic Practice, 2017.

Thornton, D. 2010. Scoring guide for Risk Matrix 2000.10/SVC. Unpublished document. 
Final peer reviewed manuscript accepted in Journal of Forensic Practice, 2017.

\section{TABLES AND FIGURES}

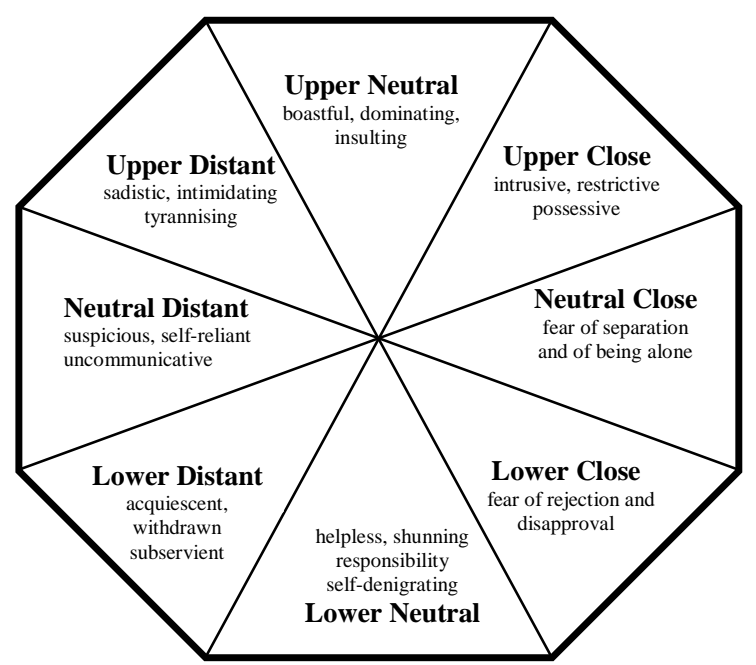

Figure 1

Characteristics of Negative Relating 
Final peer reviewed manuscript accepted in Journal of Forensic Practice, 2017.

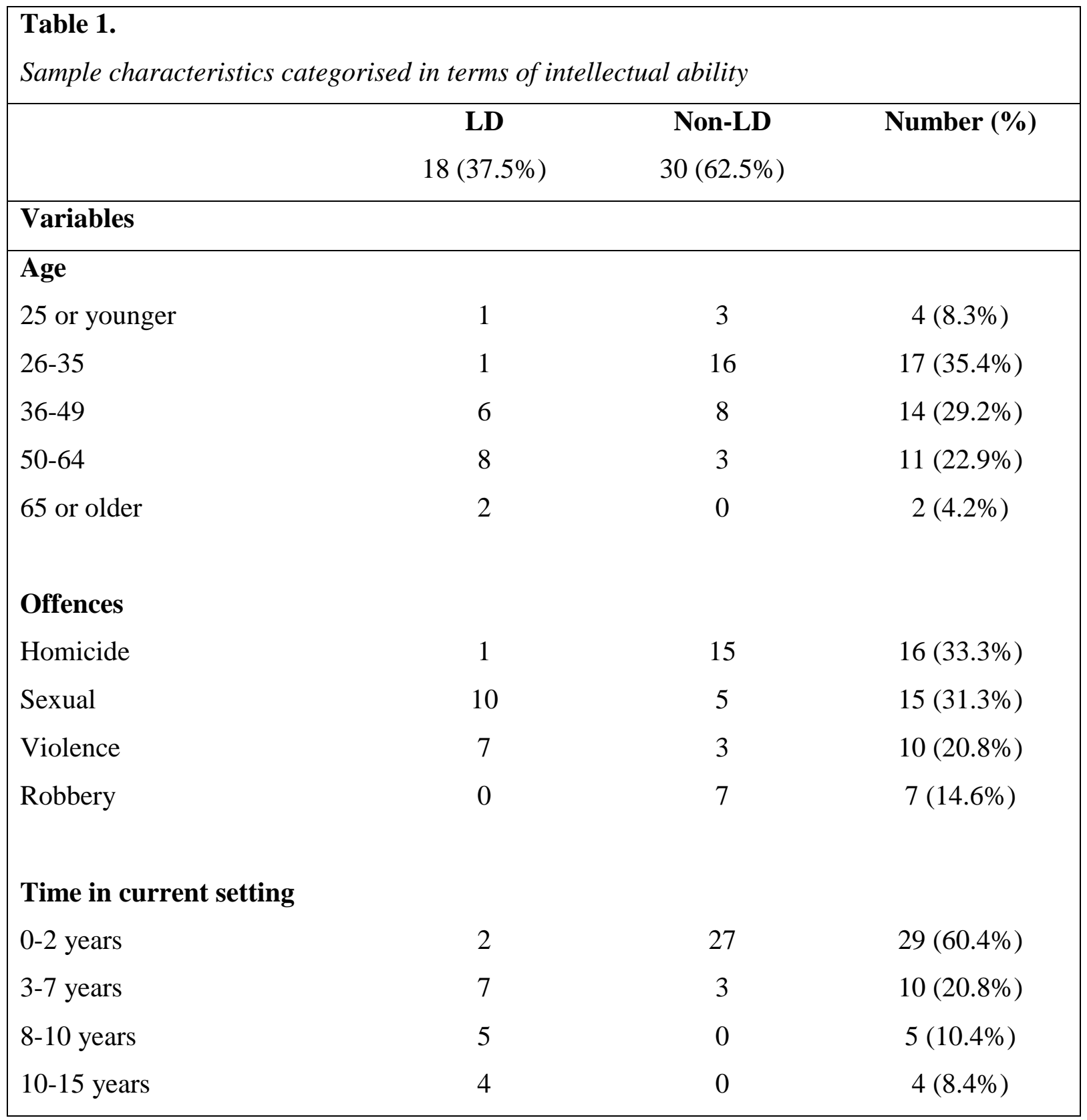


Final peer reviewed manuscript accepted in Journal of Forensic Practice, 2017.

\begin{tabular}{|c|c|c|c|c|c|}
\hline \multirow[b]{2}{*}{ PROQ3 } & \multicolumn{2}{|c|}{ LD Offenders } & \multicolumn{2}{|c|}{ Non LD Offenders } & \multirow[t]{2}{*}{$t$} \\
\hline & $\mathrm{M}$ & SD & $\mathrm{M}$ & SD & \\
\hline Upper Neutral (UN) & 8.94 & 3.64 & 4.17 & 2.96 & $4.97 * * *$ \\
\hline Upper Close (UC) & 7.44 & 4.72 & 2.47 & 2.70 & $4.09 * * *$ \\
\hline Neutral Close (NC) & 7.72 & 4.08 & 3.20 & 3.26 & $4.22 * * *$ \\
\hline Lower Close (LC) & 6.83 & 3.35 & 5.87 & 3.68 & .91 \\
\hline Lower Neutral (LN) & 6.39 & 4.41 & 5.33 & 3.45 & .92 \\
\hline Lower Distant (LD) & 10.06 & 3.04 & 5.40 & 3.45 & $4.73^{* * *}$ \\
\hline Neutral Distant (ND) & 10.22 & 3.14 & 7.67 & 3.91 & 2.35 \\
\hline Upper Distant (UD) & 7.72 & 3.82 & 4.97 & 3.23 & 2.67 \\
\hline Total Score & 65.33 & 18.53 & 39.07 & 16.89 & $5.03 * * *$ \\
\hline
\end{tabular}

Note. A Bonferroni-adjusted alpha of $p<.006$ was used.

$* * * p<.001$ 
Final peer reviewed manuscript accepted in Journal of Forensic Practice, 2017.

\begin{tabular}{|lccccc|}
\hline $\begin{array}{l}\text { Table } 3 \\
\text { Adjusted Mean for PROQ3 scores } \\
\text { functioning }\end{array}$ & \multicolumn{5}{l}{ across offence categories controlling for level of } \\
\hline \multicolumn{7}{l}{ Sexual } & Violent & Robbery & Homicide & F \\
PROQ3 & Offenders & Offenders & Offenders & Offenders & \\
\cline { 2 - 6 } Upper Neutral (UN) & 5.23 & 6.05 & 7.88 & 5.75 & $17.74^{* * *}$ \\
Upper Close (UC) & 4.52 & 4.70 & 4.30 & 3.95 & $9.96^{* *}$ \\
Neutral Close (NC) & 5.17 & 4.75 & 6.81 & 3.90 & $9.93^{* *}$ \\
Lower Close (LC) & 6.31 & 5.60 & 5.46 & 6.88 & .73 \\
Lower Neutral (LN) & 5.78 & 7.28 & 4.03 & 5.46 & .00 \\
Lower Distant (LD) & 7.48 & 8.33 & 4.21 & 7.37 & $8.10^{* *}$ \\
Neutral Distant (ND) & 7.31 & 8.11 & 10.06 & 9.55 & $7.46^{* *}$ \\
Upper Distant (UD) & 5.36 & 5.27 & 8.85 & 5.81 & $8.01^{* *}$ \\
Total Score & 47.15 & 50.08 & 51.60 & 48.67 & $14.40^{* * *}$ \\
\hline
\end{tabular}

Note. Comparisons based upon ANCOVA adjusted means controlling for the presence of a learning disability and length of detainment.

$* * p<.01 ; * * * p<.001$ 